30 days were reported by $15 \%$ and $7 \%$ of clients, respectively. Reported history of an STI was also common at $29 \%$. A previous HIV test was reported by 74\%; active/potential bridgers were significantly less likely than unlikely bridgers to have ever been tested for HIV.

Conclusions Results of this pilot study demonstrate the feasibility of successfully recruiting clients of FSW in a setting of epidemic growth and high HIV prevalence among FSW. A majority of clients of FSW also have non-FSW sex partners, and these partnerships are often concurrent. Condom use is sub-optimal with both FSW and non-FSW partners, and drug and alcohol use were common. These risk behaviours signal potential for HIV/STI transmission among male clients of FSW in St. Petersburg Russia, and indicate a pressing need to better understand the context and nature of risk for this potentially important bridging population. participants). Bridge clients were significantly less educated, more employed, paid lower prices to the FSW just visited, and had a previous STI.

Conclusions Clients showed high-risk sexual behaviour, a relatively high prevalence of HIV in clients compared to national estimates and a substantial proportion of them act as a bridge for HIV/STI transmission between FSW and the general population in Escuintla. Given that this is fuelling the current HIV epidemic, preventive interventions addressing this hard-to-reach group are urgently required. A significant challenge is to overcome the difficulty of reaching clients as well as to address the variety of high-risk behaviours among clients by partner type in this setting and take into account traditional concepts of masculinity.

\section{P1-S2.07 STI RATES AND RISK FACTORS AMONG FEMALE SEX WORKERS ATTENDING STI TESTING SITES IN GERMANY, JANUARY-DECEMBER 2010}

doi:10.1136/sextrans-2011-050108.64 BEHAVIOURS AMONG CLIENTS OF SEX WORKERS IN GUATEMALA - ARE THEY A BRIDGE IN HIV TRANSIMISSION?

doi:10.1136/sextrans-2011-050108.63

\begin{abstract}
${ }^{1,2} \mathrm{M}$ Sabidó, ${ }^{3,4} \mathrm{M}$ Lahuerta, ${ }^{5} \mathrm{G}$ Hernández, ${ }^{2,6} \mathrm{~A}$ Montoliu, ${ }^{2,6} \mathrm{~V}$ Gonzalez, ${ }^{7} \mathrm{~F}$ Giardina, ${ }^{5} \mathrm{~J}$ E Monzón, ${ }^{8} \mathrm{M}$ I Pedroza, ${ }^{2,6} \mathrm{~J}$ Casabona, ${ }^{8} \mathrm{R}$ Guevara. ${ }^{1}$ Fundació Sida i Societat, Barcelona, Catalonia, Spain; ${ }^{2}$ CIBER Epidemiología y Salud Pública (CIBERESP), Barcelona, Spain; ${ }^{3}$ International Center for AIDS Care and Treatment Programs, Columbia University-Mailman School of Public Health, New York, USA; ${ }^{4}$ Fundació Sida i Societat, Barcelona, USA; ${ }^{5}$ Fundació Sida i Societat, Escuintla, Guatemala, Guatemala; ${ }^{6}$ Centre for Epidemiological Studies on HIVIAIDS and STI of Catalonia (CEEISCAT), ICO/Health Department, Generalitat de Catalunya, Badalona, Spain; ${ }^{7}$ Fundació Sida i Societat, Barcelona, Catalonia, Spain, Spain; ${ }^{8}$ Health District of Escuintla, Ministry of Health, Guatemala, Guatemala
\end{abstract}

Background Clients of female sex workers (FSW) are an important target group for HIV/STI prevention. This study aimed to estimate the prevalence of HIV and other sexually transmitted infections (STI) prevalence in clients examine their risk behaviours and evaluate their role as a bridge population in the spread of HIV/STI.

Methods Cross-sectional study conducted between 2008 and 2009 among 553 clients recruited through a two-stage convenient sample in commercial sex sites in the province of Escuintla, Guatemala. They were interviewed and tested for HIV and other STI. Bridge clients were defined as those who engaged in sex with both regular partners and FSWs but reported inconsistent condom use with FSWs whereas potential bridge as those reported consistent condom use with FSWs. Non-bridge clients engaged in sex with only FSWs. Multinomial and multivariable logistic regression models were used to identify risk factors associated with the bridge category and with having $\geq 1$ STI/HIV, respectively.

Results Half of the clients approached refused participation. Median age was 28.9 years; $57.7 \%$ had a regular partner, of whom $10.1 \%$ had concurrent non-commercial partnerships. Consistent condom use with FSW and regular partners was $72.5 \%$ and $17.1 \%$, respectively. The main reason for not using condoms was trust (49.5\%). Approximately $18 \%$ formed a bridge, and $40.0 \%$ a potential bridge. Among those who provided samples $(70.5 \%$ a blood sample and $89.7 \%$ urine sample), prevalence of HIV, syphilis, gonorrhoea, chlamydia, and herpes simplex virus 2 was $1.5 \%, 1.0 \%, 0.8 \%, 5.5 \%$, and $3.4 \%$, respectively. Unprotected sex with FSWs and drug use just before sex were risk factors for having any STI $(9.8 \%$ of 
Abstract P1-S2.07 Table 1 Association between selected risk factors and being "STI positive" (testing positive for Chlamydia, Gonorrhoea, Syphilis and/or Trichomoniasis) among female sex workers attending STI testing sites in Germany, $2010(\mathrm{~N}=1142)$

\begin{tabular}{|c|c|c|c|c|}
\hline & \multirow[b]{2}{*}{$\mathbf{N}_{\text {Total }}$} & \multicolumn{3}{|c|}{$\begin{array}{l}\text { STI positive (Chlamydia, Gonorrhoea, } \\
\text { Syphilis and/or Trichomoniasis), } \mathrm{N}=\mathbf{2 3 3}\end{array}$} \\
\hline & & $\overline{N_{\text {Positive }}}$ & OR $(95 \% \mathrm{Cl})$ & $\mathrm{p}$ Value \\
\hline \multicolumn{5}{|l|}{ German language } \\
\hline Fluent (R) & 437 & 59 & 1 & \\
\hline Medium & 301 & 34 & $0.82(0.52$ to 1.30$)$ & 0.376 \\
\hline Very poor / None & 375 & 135 & $3.60(2.55$ to 5.10$)$ & 0.000 \\
\hline \multicolumn{5}{|l|}{ Age } \\
\hline Range $15-77$ years & 1142 & 233 & $0.95(0.93$ to 0.97$)$ & 0.000 \\
\hline \multicolumn{5}{|l|}{ Health insurance } \\
\hline No (R) & 450 & 138 & 1 & \\
\hline Yes & 648 & 87 & $0.35(0.26$ to 0.47$)$ & 0.000 \\
\hline \multicolumn{5}{|c|}{ Meet clients on the street } \\
\hline No (R) & 995 & 173 & 1 & \\
\hline Yes & 101 & 43 & $3.52(2.30$ to 5.40$)$ & 0.000 \\
\hline \multicolumn{5}{|c|}{ Meet clients through internet or adds } \\
\hline No (R) & 908 & 195 & 1 & 0.002 \\
\hline Yes & 188 & 21 & $0.46(0.28$ to 0.74$)$ & \\
\hline \multicolumn{5}{|c|}{ Number of clients per week } \\
\hline Range $1-75$ & 270 & 37 & $1.02(1.00$ to 1.04$)$ & 0.028 \\
\hline
\end{tabular}

Explanatory factor included as continuous variable in logistic regression.

(R), Reference group.

\section{P1-S2.08 GEOGRAPHY, MOBILITY, WORK VENUE, AND SEXUALLY TRANSMITTED INFECTIONS AMONG 4100 FEMALE SEX WORKERS IN 20 CITIES IN PERU}

doi:10.1136/sextrans-2011-050108.65

${ }^{1} \mathrm{C}$ Mejia, ${ }^{2} \mathrm{P}$ Campos, ${ }^{2} \mathrm{C}$ Carcamo, ${ }^{2} \mathrm{P}$ Garcia, ${ }^{3} \mathrm{~J}$ Hughes, ${ }^{3} \mathrm{D}$ Martin, ${ }^{4} \mathrm{G}$ Garnett, ${ }^{3} \mathrm{~K}$ Holmes. ${ }^{1}$ Battelle, Seattle, USA; ${ }^{2}$ Universidad Peruana Cayetano, Peru; ${ }^{3}$ University of Washington, Seattle, USA; ${ }^{4}$ mperial College, UK

Objectives To estimate and compare prevalences of five STI Chlamydia trachomatis (CT) Neisseria gonorrhoeae (NG), Trichomonas vaginalis (TV), T pallidum (syphilis), and HIV infection for female sex workers (FSW) according to home geographic region and mobility, and to identify other STI risk factors.

Methods Data on geographic region, internal mobility (defined as having engaged in sex work in $>2$ Peruvian cities in the previous 12 months or was working away from their home city at enrolment), sex work characteristics, sociodemographics and, behaviours, were collected together with vaginal and blood specimens from 4100 FSW in 20 Peruvian cities. Multivariate analysis identified factors associated being infected with any of the five STI.

Results FSW from the jungle region had higher prevalences of CT, NG, TV, syphilis, and HIV than FSW from the coastal or highlands regions. In univariate analysis, having worked in $>2$ more cities or working away from home were significantly negatively associated with having any STI. In multivariate analyses the mobility status variable showed a trend towards protective associations; neither remained significant. Risk of any STI was significantly and independently associated with bar/nightclub or street work, being from the jungle region, and not completing high school.

Conclusion Findings suggest that internal mobility does not increase risk among Peruvian FSW. Preventive interventions should focus on FSW from the jungle region, those working in bars/ nightclubs or on the street and those lacking secondary education to halt the spread of STI among FSW and, presumably, to the general population.

\section{P1-S2.09 WHO IS AT HIGHER RISK OF STIS AND HIV-BROTHEL-BASED OR STREET-BASED FEMALE SEX WORKERS? EVIDENCE FROM TWO ROUNDS OF BIO-BEHAVIOURAL SURVEYS}

doi:10.1136/sextrans-2011-050108.66

${ }^{1}$ A Gautam, ${ }^{1} \mathrm{R}$ Adhikary, ${ }^{1} \mathrm{~S}$ Ramanathan, ${ }^{1} \mathrm{P}$ Goswami, ${ }^{1} \mathrm{~S}$ Khobragade, ${ }^{2} \mathrm{~S} M$ Deshpande, ${ }^{2} \mathrm{~S}$ Khazi, ${ }^{2} \mathrm{M}$ M Mainkar, ${ }^{2} \mathrm{R}$ S Paranjape. ${ }^{1} \mathrm{FHI}$, New Delhi, India, ${ }^{2}$ National AIDS Research Institute, NARI, India

Background Female sex workers (FSWs) are considered a high-risk group for HIV and other STIs. Sex work practices of FSWs in Maharashtra are diverse and vary according to the sex work milieu (eg, street-, brothel- and bar-based). We examined changes in HIV and STI prevalence, associated risk behaviours and socio-economic variables among street-based (SB) and brothel-based (BB) FSWs in Maharashtra.

Methods Two rounds of cross-sectional surveys were conducted in 2005 (R1) and 2009 (R2) in selected districts of Maharashtra. Probability sampling methods, such as conventional and time-location cluster sampling, were used to sample 2257 (1212 BB and 1045 SB) FSWs in R1 and 2228 (1182 BB and 1046 SB) FSWs in R2. Sexual behaviours were assessed, blood and urine samples were collected to measure the prevalence of HIV, Neisseria gonorrhoeae (NG), Chlamydia trachomatis (CT) and syphilis. Changes were analysed using bivariate and multivariate methods.

Results HIV prevalence increased significantly among SB FSWs from $17.2 \%$ in $\mathrm{R} 1$ to $24.3 \%$ in $\mathrm{R} 2$ (OR $1.54, \mathrm{p}=0.04$ ), whereas among $\mathrm{BB}$ FSWs, there was no significant change (28.4 in R1 to 28.9 in R2, OR $1.03, \mathrm{p}=0.88$ ). Prevalence of active syphilis (TPHA and RPR positive at any titre) declined among BB FSWs from $15.4 \%$ (R1) to $11.0 \%$ (R2) (OR 0.67, $\mathrm{p}=0.05$ ), while the decline among SB FSW was not significant (R1-13.6\% to R2-10.6\%; OR 0.75, $\mathrm{p}=0.22$ ). NG prevalence fell from $7.7 \%$ (R1) to $3.9 \%$ (R2) (OR 0.48, $\mathrm{p}=0.006$ ) for $\mathrm{BB}$ FSWs, whereas for SB FSWs, it declined from $7.2 \%$ in R1 to $4.2 \%$ in R2 (OR 0.56, $\mathrm{p}=0.07$ ). No significant change was observed in the prevalence of CT in either group. Self-perceived risk of HIV among BB FSWs increased from $52.5 \%$ (R1) to $62.5 \%$ (R2), (OR 1.5, $\mathrm{p}=0.02)$; whereas among SB FSWs, it was unchanged (49.8\% in R1 to $50.3 \%$ in $\mathrm{R} 2$; OR $1.02, \mathrm{p}=0.23)$. The proportion of illiterate $\mathrm{BB}$ FSWs decreased from $80.1 \%$ (R1) to $74.3 \%$ (R2), (OR $0.72, p=0.02$ ) while for SB FSWs, illiteracy increased significantly from $64.4 \%$ (R1) to $77.1 \%$ (R2), (OR 1.86, $\mathrm{p}<0.001$ ). The proportion of FSWs having more than 10 clients per week was same over both rounds for BB FSWs but for SB FSWs, it increased significantly to $49.9 \%$ (R2) from $32.8 \%$ (R1) (OR 2.04, p<0.001).

Conclusion SB FSWs are at higher risk of contracting HIV and other STIs and are more vulnerable compared to BB FSWs. Prevention interventions must account for differences in profiles, risk behaviours and sex work typology to customise strategies for these groups for maximum impact.

\section{P1-S2.10 FEMALE CLIENTS AND PARTNERS OF MSM SEX WORKERS IN A RESOURCE-POOR SETTING, WEST AFRICA}

doi:10.1136/sextrans-2011-050108.67

${ }^{1} 0$ Busari, ${ }^{2} \mathrm{M}$ Nakayima, ${ }^{3} \mathrm{~A}$ Busari. ${ }^{1}$ Federal Medical Centre, Ido-Ekiti, Nigeria; ${ }^{2}$ The AIDS Support Organization, Masaka, Uganda; ${ }^{3}$ Care AIDS International, Nigeria

MSM are becoming increasingly important in HIV epidemics in Africa and their population is growing in Nigeria. However, there's paucity of data on HIV epidemics in MSM in Nigeria and West Africa region. Though HIV transmission in Africa is mainly heterosexual, interactions between HIV epidemics in MSM and 